\title{
Effect of hypertension and its reverse on serum nitric oxide concentration and vascular permeability in two-kidney one-clip hypertensive rats
}

\author{
Majid Khazaei ${ }^{1}$, Mohammad Zarei ${ }^{2}$, Mohammad R. Sharifi ${ }^{1}$ and Ali A. Pourshanazari ${ }^{1}$ \\ ${ }^{1}$ Department of Physiology, Faculty of Medicine, Isfahan University of Medical Sciences, Isfahan, Iran \\ ${ }^{2}$ Department of Physiology, Faculty of Medicine, Hamadan University of Medical Sciences, Hamadan, Iran
}

\begin{abstract}
The aim of this study was to evaluate the effect of hypertension and its reverse on serum nitric oxide (NO) concentration and endothelial permeability in two-kidney one-clip (2K1C) hypertensive rats. 28 male Wistar rats were divided into four groups: 1) 2K1C for 12 weeks; 2) sham-clipped for 12 weeks; 3) 2K1C for 12 weeks and unclipped for 12 weeks; 4 ) sham-clipped for 12 weeks and unclipped for 12 weeks. Blood samples were taken before experiment, $12^{\text {th }}$ week and $24^{\text {th }}$ week (in groups 3 and 4 ). Coronary vascular and aortic endothelial permeability were determined by extravasation of Evans blue dye method. Serum NO level was significantly lower in hypertensive group compare with sham group $(4.21 \pm 1.28 v s .9 .47 \pm 1.34 \mu \mathrm{mol} / \mathrm{l}$, respectively). Reversal of hypertension did not improve serum NO concentration in $2 \mathrm{~K} 1 \mathrm{C}$ group $(4.21 \pm 1.28$ vs. $4.32 \pm 1.34 \mu \mathrm{mol} / \mathrm{l})$. Coronary vascular and aortic endothelial permeability were not different between hypertensive and normotensive groups and reversal of hypertension did not alter endothelial permeability. Lower serum $\mathrm{NO}$ concentration in $2 \mathrm{~K} 1 \mathrm{C}$ hypertensive rats even after reversal of hypertension suggested that in addition to NO, other mechanisms could be involved in surgical reversal of hypertension. Hypertension and its reverse did not change endothelial permeability at least in this model of hypertension.
\end{abstract}

Key words: Hypertension — Two-kidney one-clip — Nitric oxide - Permeability — Endothelium

Abbreviations: 2K1C, two-kidney one-clip; EB, Evans blue; EP, endothelial permeability; PRA, plasma renin activity; SBP, systolic blood pressure.

\section{Introduction}

Hypertensive patients are at particular risk of cardiovascular complications and stroke, possibly related to endothelial dysfunction (Viazzi et al. 2008). Endothelium has an essential role in control of vascular function and homeostasis. It is believed that endothelial dysfunction is a preclinical stage of atherosclerosis processes and considered as a target for cardiovascular risk factors (Feletou and Vanhoutte 2006). Endothelial dysfunction during hypertension leads to dysregulation of many important vascular functions including

Correspondence to: Majid Khazaei, Department of Physiology, Faculty of Medicine, Isfahan University of Medical Sciences, Isfahan, Iran

E-mail: Khazaei@med.mui.ac.ir platelet aggregation or leukocyte adhesion (Khazaei et al. 2008; Versari et al. 2009).

The vascular endothelium is capable of synthesizing nitric oxide (NO) from L-arginine. $\mathrm{NO}$ as a biological messenger and effector molecule is known to be involved in several physiological and pathophysiological processes in various organ systems (Bauer and Sotnikova 2010) and had been implicated in the pathogenesis of several diseases including hypertension (Hermann et al. 2006; Torok 2008). Studies have provided evidences that impairment of NO production accounts for the abnormalities in vascular function in hypertension in animals and humans (Hermann et al. 2006; Bian et al. 2008; Torok 2008). Several studies have shown that NO is closely involved in the regulation of vascular tone and systemic blood pressure. In addition, NO has several antiatherosclerotic 
properties including inhibition of platelet aggregation, leukocyte adhesion to endothelial cells, smooth muscle cell proliferation and migration (Ross 1999; Kawashima and Yokoyama 2004).

Hypertension is a risk factor for cardiovascular disease and recent studies suggested that increased endothelial permeability may have a role in development of end organ damage (Viazzi et al. 2008). Furthermore, it is believed that endothelial cell injury is responsible for the development of atherosclerosis (Luscher 1994; Torok 2008). Changes in injured endothelium lead to disruption of its permeability characteristics and increase retaining of oxidised LDL in subintimal space which initiates a vicious cycle for atherosclerosis (Ross 1993, 1999). Therefore, we hypothesize that chronic hypertension alters vascular endothelial permeability and serum NO concentration as a well known marker of endothelial function in two-kidney one-clip (2K1C) hypertensive rats. $2 \mathrm{~K} 1 \mathrm{C}$ hypertensive model is a well known model of hypertension. Increased activity of renin-angiotensin-aldosterone system is responsible for rising blood pressure in this model especially in first 6 weeks (MartinezMaldonado 1991; Nystrom et al. 2002) and subsequent changes of vascular structure is important in maintenance of hypertension (Martinez-Maldonado 1991). In the second part of this study, we tried to assess changes of endothelial permeability and serum NO concentration after surgical reversal of hypertension to evaluate whether the changes are reversible after blood pressure reduction.

\section{Materials and Methods}

\section{Animals and experimental groups}

28 male Wistar rats with an initial body weight of 180 $200 \mathrm{~g}$ were purchased from Pasteur Institute of Iran. The animals were housed in cages ( 3 or 4 per cage) at $22-25^{\circ} \mathrm{C}$ with $12 \mathrm{~h}$ light/dark cycle. The animals were randomly divided into two groups: sham-clipped and $2 \mathrm{~K} 1 \mathrm{C}$ hypertensive rats. After surgery, each group was divided into two subgroups: clipping and unclipping groups. The experimental groups were as follow:

group 1: $2 \mathrm{~K} 1 \mathrm{C}$ for 12 weeks $(n=7)$

group 2: sham-clipped for 12 weeks $(n=7)$

group 3: $2 \mathrm{~K} 1 \mathrm{C}$ for 12 weeks and unclipped for 12 weeks $(n=7)$

group 4: sham-clipped for 12 weeks and unclipped for 12 weeks $(n=7)$.

Blood samples were taken from all animals on pre-clipping, $12^{\text {th }}$ week and $24^{\text {th }}$ week (in groups 3 and 4 ). Samples were centrifuged and plasma and serums were kept in $-70^{\circ} \mathrm{C}$ for further analysis. All procedures were conducted and approved from ethical committee of Isfahan University of
Medical Sciences in accordance with guideline for care and use of laboratory animals.

\section{Preparation of $2 \mathrm{~K} 1 \mathrm{C}$ hypertensive rats}

Rats were anesthetized with intraperitoneally injection of ketamine hydrochloride $(60 \mathrm{mg} / \mathrm{kg})$ and xylazine $(7.5 \mathrm{mg} / \mathrm{kg})$ and underwent left renal artery constriction with a silver clip (internal gap of $0.20 \mathrm{~mm}$ ) as described previously (Kunes 1993; Diekmann et al. 2000; Nystrom et al. 2002). In sham-clipped group, the same procedure was done without using silver clip. After closing the wound, penicillin G (25000 U i.m) was injected. All rats were fed with commercial rat chow (Pasteur Institute, Iran) and allowed free access to tap water during the experiments.

\section{Reversal of 2K1C hypertension}

In groups 3 and 4 , unclipping was carried out on $12^{\text {th }}$ weeks (12 weeks after clipping). For this purpose, the rats were anesthetized. Through a flank incision, the clipped left renal artery was exposed and fibrous tissues surrounding the clip and renal artery were carefully dissected. Then, the clip was removed gently. In sham-unclipped group, the same procedure was done without removing the clip.

\section{Blood pressure measurement}

During the study, systolic blood pressure (SBP) was measured under conscious conditions twice a week by tail-cuff method with non-invasive blood pressure controller PowerLab software (ADInstruments Company, USA). Before sacrificing rats, direct blood pressure was also measured by cannulation of the carotid artery (PE-50) using an invasive blood pressure controller PowerLab software.

\section{Measurement of serum NO concentration}

Serum NO concentrations were measured by Griess reagent system (Promega Corporation, Madison, USA), using available reagents as previously described (Khazaei and Nematbakhsh 2006). In brief, serums were added into wells (96-well enzymatic assay plate). Sulfanilamide solution was added to all collected samples and then $N$-1-naphtylethylenediamine dihydrochloride under acidic conditions was added. The absorbance was detected by a microplate reader in $520-550 \mathrm{~nm}$ wavelengths. The samples NO concentration was determined by comparison to nitrite standard curve. The limit detection was $2.5 \mu \mathrm{M}$ nitrite.

\section{Measurement of endothelial permeability}

Coronary vascular and aortic endothelial permeability were determined by extravasation of Evans blue (EB) dye method 
as previously described (Hulthen et al. 1996; Khazaei and Nematbakhsh 2004). First, EB (diluted in normal saline; 20 $\mathrm{mg} / \mathrm{ml}$ ) was injected through the carotid artery cannula. After $20 \mathrm{~min}$, rats were sacrified. Heart and aorta (ascending aorta to the origin of renal arteries branches) were isolated and cleaned from surrounding connective tissues. Then, they were weighed and put into formamide solution overnight at room temperature for EB dye extraction. The extracted amount of EB in formamide solution was determined by spectrophotometer (Secomam, France) at $620 \mathrm{~nm}$ wavelength. The results were plotted on standard of EB in 0.2 to $10 \mu \mathrm{g} / \mathrm{ml}$ formamide. Concentration of EB in these tissues was expressed in $\mu \mathrm{g} / \mathrm{g}$ wet weight $(\mu \mathrm{g} / \mathrm{g}$ ww) tissue.

\section{Plasma renin activity}

Plasma renin activity (PRA) was measured using I-125 Angiotensin I generation. Angiotensin I coated-tube radioimmunoassay was performed in two aliquots of the same sample, one incubated at $37^{\circ} \mathrm{C}$ for generation and one nonincubated. PRA was calculated as nanogram angiotensin I generated/ml/h (Renctk P2721, DiaSorin Biomedica Diagnostic Division RIA kit, Italy). The PRA assay sensitivity was $0.13 \mathrm{ng} / \mathrm{ml}$; intra-and interassay coefficients of variation were 7.5 and $7.7 \%$, respectively.

\section{Statistical analysis}

Data are expressed as mean \pm SEM. Comparison of data in the same group before and after intervention were made using the paired $t$-test. Comparisons of data among groups were carried out using one-way ANOVA. $p<0.05$ was considered statistically significant.

\section{Results}

\section{Blood pressure}

SBP values after clipping and unclipping in all experimental groups are shown in Fig. 1 which illustrates higher SBP of 2K1C group compare to sham-clipped group (162.84 \pm 3.79

Table 1. Plasma renin activity (PRA) values in $2 \mathrm{~K} 1 \mathrm{C}$ hypertensive rats

\begin{tabular}{lcc}
\hline Rats & $n$ & PRA (ng/ml/h) \\
\hline Pre-clipping & 11 & $3.14 \pm 0.18$ \\
Post-clipping & 14 & $5.70 \pm 0.24^{*}$ \\
Unclipping & 7 & $3.32 \pm 0.16$ \\
\hline
\end{tabular}

Data are expressed as mean \pm S.E. ${ }^{*} p<0.05$ compare to other groups.

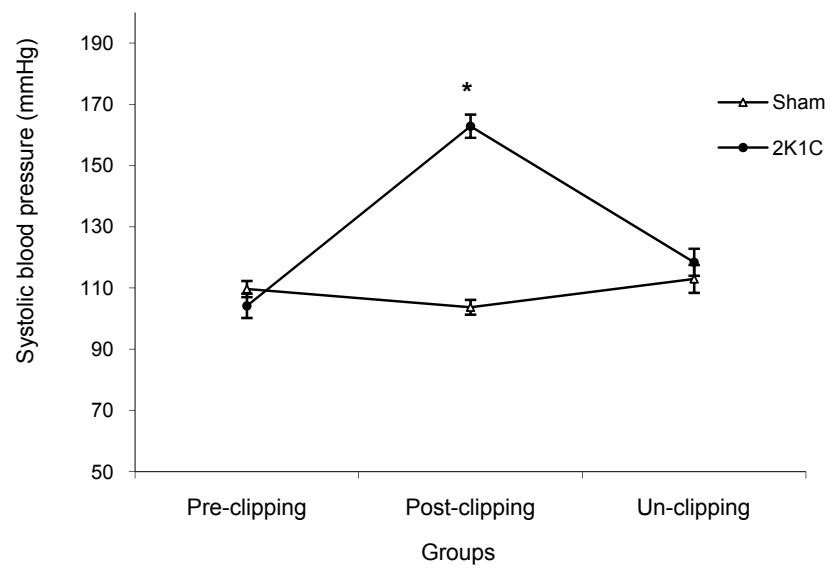

Figure 1. Systolic blood pressure values in pre-clipping, post-clipping and unclipping rats. ${ }^{*} p 0.05 v s$. other groups.

$v s .104 .16 \pm 3.96 \mathrm{mmHg} ; p<0.05)$. After unclipping, SBP of $2 \mathrm{~K} 1 \mathrm{C}$ hypertensive rats significantly reduced and reached to sham-unclipped level $(162.84 \pm 3.79 v s .118 .4 \pm 4.42$ $\mathrm{mmHg}, p<0.05)$.

\section{PRA measurement}

PRA levels were measured in $2 \mathrm{~K} 1 \mathrm{C}$ hypertensive rats and are shown in Table 1. PRA level was significantly higher in $2 \mathrm{~K} 1 \mathrm{C}$ after clipping and reduced after unclipping $(p<0.05)$.

\section{Serum NO concentration}

Fig. 2 illustrates serum NO concentration in sham and 2K1C hypertensive rats before and after clipping and unclipping. There was no significant difference in serum NO concentration in pre-clipping between groups $(p>0.05)$. After clipping, serum NO concentration in $2 \mathrm{~K} 1 \mathrm{C}$ hypertensive rats was

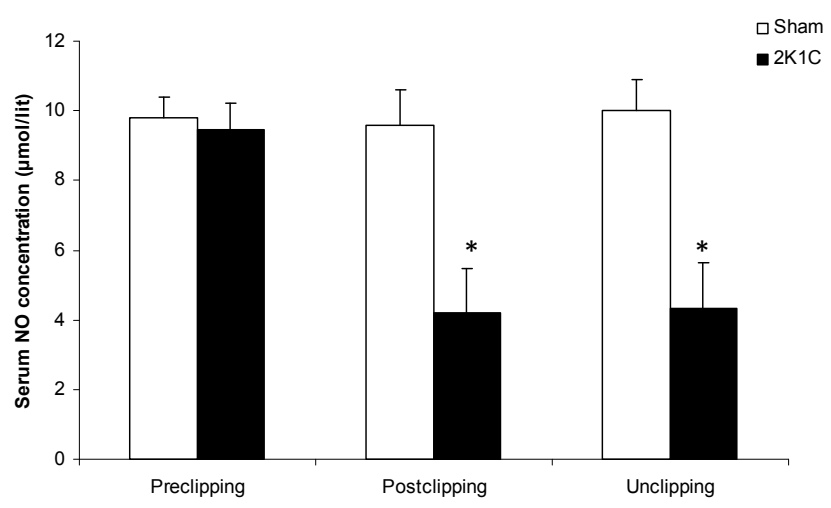

Figure 2. Serum NO concentration in sham and $2 \mathrm{~K} 1 \mathrm{C}$ hypertensive rats before and after clipping and unclipping ( $n=7$ each group). ${ }^{*} p<0.05$ vs. sham group. 
decreased and it was significantly lower than sham-clipped group $(p<0.05)$. Unclipping and surgical reversal of hypertension in $2 \mathrm{~K} 1 \mathrm{C}$ group did not improve serum $\mathrm{NO}$ level and it was lower than sham-unclipped group $(p<0.05)$.

\section{Vascular endothelial permeability}

Vascular endothelial permeability of aorta (expressed as quantitative extravasations of $\mathrm{EB}$ ) in $2 \mathrm{~K} 1 \mathrm{C}$ hypertensive group was lower than sham-clipped group, although it was not statistically significant $(p>0.05)$ (Table 2$)$. Unclipping didn't alter aortic endothelial permeability in $2 \mathrm{~K} 1 \mathrm{C}$ group $(p>0.05)$. Also, vascular endothelial permeability of coronary circulation wasn't different between $2 \mathrm{~K} 1 \mathrm{C}$ and shamclipped group and didn't alter after unclipping $(p>0.05)$.

\section{Discussion}

Endothelium is an early target of cardiovascular risk factors such as hypertension. Endothelial dysfunction is characterized by changes in endothelial permeability, endothelium-derived factors and vasomotor responses. Among the endothelium-derived vasoactive factors, NO is one of the most well characterized. In this study, we found that serum $\mathrm{NO}$ concentrations in $2 \mathrm{~K} 1 \mathrm{C}$ hypertensive rats were lower than sham-clipped group. Impaired endothelium-dependent relaxation indicated in all models of hypertension including 2K1C (Luscher and Vanhoutte 1986; Nakamura and Prewitt 1992) and in human hypertension (Linder et al. 1990). In this study, we measured serum NO level after 12 weeks induction of hypertension. It is indicated that after renal artery clipping, NO synthesis increases during two weeks and then gradually declines during next weeks (Dubey et al. 1996). The later decrease in serum NO level could be the result of either endothelial dysfunction due to hypertension or adaptation to high blood pressure. NO has several beneficial role in vascular homeostasis including vascular tone, control of blood pressure and antiatherosclerotic properties (Bian et al. 2008; Pepine 2009) and decreased NO bioavailability could explain why cardiovascular risk factors such as

Table 2. Quantitative extravasations of aorta and heart Evans blue (EB)

\begin{tabular}{llccc}
\hline & Group & $n$ & $\begin{array}{c}\text { Aorta EB } \\
(\mu \mathrm{g} / \mathrm{g} w w)\end{array}$ & $\begin{array}{c}\text { Heart EB } \\
(\mu \mathrm{g} / \mathrm{g} w w)\end{array}$ \\
\hline \multirow{2}{*}{ Post-clipping } & Sham & 7 & $33.72 \pm 3.58$ & $17.62 \pm 1.40$ \\
& 2K1C & 7 & $24.58 \pm 1.62$ & $12.57 \pm 1.65$ \\
\cline { 2 - 5 } Unclipping & Sham & 7 & $32.94 \pm 6.19$ & $14.19 \pm 3.68$ \\
& 2K1C & 7 & $25.84 \pm 3.89$ & $15.95 \pm 1.76$ \\
\hline
\end{tabular}

Data are expressed as mean \pm S.E. ww, wet weight. hypertension are considered risk factor for atherosclerosis. $\mathrm{NO}$ also affect endothelial permeability in different tissues, although there are contradictory reports (Filep and FoldesFilep 1993; Yuan et al. 1993). Some studies reported that NO synthase inhibitor increased microvascular permeability in stomach, intestine, liver, spleen, pancreas, kidney, and lung (Filep and Foldes-Filep 1993) whereas in other studies, NO inhibitor decreased microvascular permeability of venules (Yuan et al. 1993)

Decreased NO bioavailability in hypertensive subjects may results from lower NO production such as deficiency in L-arginine/BH4 (Cosentino and Luscher 1999; Zhou et al. 2001) or increased NO degradation due to higher superoxide anion generation or reduced level of antioxidant (Schulman et al. 2005). Furthermore, 2K1C goldblatt model induces renin-angiotensin dependent hypertension and another explanation for reduced NO availability in this model of hypertension is that high angiotensin II level decreases NO bioavailability by promoting oxidative stress (de Gasparo 2002).

We also found that surgical reversal of hypertension did not improve serum NO concentration while blood pressure reached to normotensive level. In agreement to our results, a study in renovascular hypertensive rats revealed that production of NO partly contributes to blood pressure reduction after unclipping (Huang and Tsai 1998). Thus, it seems that in addition to NO, other mechanisms could be involved in surgical reversal of hypertension.

The barrier function of endothelium and control of its permeability that regulates macromolecular transport is a key defense mechanism against vascular injury and atherosclerosis (van Hinsbergh 1997; De Caterina 2000). In addition, recent studies suggested that increased vascular endothelial permeability have a role in development of end organ damage during hypertension (Viazzi et al. 2008). Thus, in this study, we expected higher endothelial permeability in $2 \mathrm{~K} 1 \mathrm{C}$ hypertensive group compare to sham animals. However, we did not find any difference in endothelial permeability between these groups. It seems that elevated blood pressure and disruption of the starling equilibrium could increase endothelial permeability (Parving and Gyntelberg 1973); however, in this study, preservation of endothelial permeability in $2 \mathrm{~K} 1 \mathrm{C}$ hypertensive group indicated that change of hemodynamic forces in hypertension does not contribute in endothelial permeability (Pedrinelli et al. 1999). In agreement to our results, a recent clinical study evaluated systemic capillary permeability using TERalb (another parameter for estimation of capillary permeability) and found that systemic capillary permeability was not different between normotensive and hypertensive group (Dell'Omo et al. 2004). In another study, TERalb was higher in atherosclerotic patients, however, was not different between normotensive and hypertensive subjects (Pedrinelli et al. 1998). In contrast, another study demonstrated that 
endothelial permeability was higher in hypertensive subjects, but endothelium-dependent relaxation was not different in uncomplicated mild to moderate hypertension (Paulis et al. 2008). We also found that reversal of hypertension did not alter endothelial permeability. Although, we do not have direct information about the vascular structural changes, it have been already shown that permeability of both media and endothelium are changed during hypertension (Kennedy and Tedgui 1995). It's suggested that changes in vascular structure during hypertension is adaptive, rather than causal (Panza et al. 1993).

In conclusion, lower serum NO concentration in $2 \mathrm{~K} 1 \mathrm{C}$ hypertensive rats even after surgical reversal of hypertension suggested that in addition to NO, other mechanisms such as bradykinin, histamine or other endothelium-derived releasing factors could be involved in surgical reversal of hypertension. Also, hypertension did not significantly change coronary vascular and aortic endothelial permeability and reversal of high blood pressure did not alter endothelial permeability.

Acknowledgment. This study was supported by Isfahan University of Medical Sciences (grant No. 187008).

\section{References}

Bauer V., Sotnikova R. (2010): Nitric oxide - the endotheliumderived relaxing factor and its role in endothelial functions. Gen. Physiol. Biophys. 29, 319-340 doi:10.4149/gpb_2010_04_319

Bian K., Doursout M. F., Murad F. (2008): Vascular system: role of nitric oxide in cardiovascular diseases. J. Clin. Hypertens. (Greenwich.) 10, 304-310 doi:10.1111/j.1751-7176.2008.06632.x

Cosentino F., Luscher T. F. (1999): Tetrahydrobiopterin and endothelial nitric oxide synthase activity. Cardiovasc. Res. 43, 274-278 doi:10.1016/S0008-6363(99)00134-0

De Caterina R. (2000): Endothelial dysfunctions: common denominators in vascular disease. Curr. Opin. Clin. Nutr. Metab Care 3, 453-467 doi:10.1097/00075197-200011000-00007

de Gasparo M. 2002: Angiotensin II and nitric oxide interaction. Heart Fail. Rev. 7, 347-358 doi:10.1023/A:1020714518246

Dell'Omo G., Penno G., Pucci L., Mariani M., Del Prato S., Pedrinelli R. (2004): Abnormal capillary permeability and endothelial dysfunction in hypertension with comorbid Metabolic Syndrome Atherosclerosis 172, 383-389

Diekmann F., Zart R., Thone-Reineke C., Bauer C., Neumayer H. H., Hocher B. (2000): Regulation of the renal endothelin system in the two-kidney, one clip renal hypertensive rat. J. Cardiovasc. Pharmacol. 36, S191-194

Dubey R. K., Boegehold M. A., Gillespie D. G., Rosselli M. (1996): Increased nitric oxide activity in early renovascular hypertension. Am. J. Physiol. 270, R118-124
Feletou M., Vanhoutte P. M. (2006): Endothelial dysfunction: a multifaceted disorder (The Wiggers Award Lecture). Am. J. Physiol. Heart Circ. Physiol. 291, H985-1002 doi:10.1152/ajpheart.00292.2006

Filep J. G., Foldes-Filep E. (1993): Modulation by nitric oxide of platelet-activating factor-induced albumin extravasation in the conscious rat. Br. J. Pharmacol. 110, 1347-1352

Hermann M., Flammer A., Luscher T. F. (2006): Nitric oxide in hypertension J. Clin. Hypertens. (Greenwich.) 8, 17-29 doi:10.1111/j.1524-6175.2006.06032.x

Huang W. C., Tsai R. Y. (1998): Nitric oxide synthesis inhibition retards surgical reversal of one-kidney Goldblatt hypertension in rats. Hypertension 32, 534-540

Hulthen U. L., Cao Z., Rumble J. R., Cooper M. E., Johnston C. I. (1996): Vascular hypertrophy and albumin permeability in a rat model combining hypertension and diabetes mellitus. Effects of calcium antagonism, angiotensin converting enzyme inhibition, and angiotensin II-AT1-receptor blockade. Am. J. Hypertens. 9, 895-901 doi:10.1016/S0895-7061(96)00177-X

Kawashima S., Yokoyama M. (2004): Dysfunction of endothelial nitric oxide synthase and atherosclerosis. Arterioscler. Thromb. Vasc. Biol. 24, 998-1005 doi:10.1161/01.ATV.0000125114.88079.96

Kennedy J. H., Tedgui A. (1995): Normal and pathological aspects of mass transport across the vascular wall. Cardiovasc. Surg. 3, 611-615 doi:10.1016/0967-2109(96)82858-4

Khazaei M., Moien-Afshari F., Laher I. (2008): Vascular endothelial function in health and diseases. Pathophysiology 15, 49-67 doi:10.1016/j.pathophys.2008.02.002

Khazaei M., Nematbakhsh M. (2004): Coronary vascular and aortic endothelial permeability during estrogen therapy: a study in DOCA-salt hypertensive ovariectomized rats. Physiol. Res. 53, 609-614

Khazaei M., Nematbakhsh M. (2006): The effect of hypertension on serum nitric oxide and vascular endothelial growth factor concentrations. A study in DOCA-Salt hypertensive ovariectomized rats. Regul. Pept. 135, 91-94 doi:10.1016/j.regpep.2006.04.003

Kunes J. (1993): The influence of age on the development of twokidney, one-clip hypertension in the rat. Physiol. Res. 42, 205-208

Linder L., Kiowski W., Buhler F. R., Luscher T. F. (1990): Indirect evidence for release of endothelium-derived relaxing factor in human forearm circulation in vivo. Blunted response in essential hypertension. Circulation 81, 1762-1767

Luscher T. F. (1994): The endothelium and cardiovascular disease--a complex relation. N. Engl. J. Med. 330, 1081-1083 doi:10.1056/NEJM199404143301511

Luscher T. F., Vanhoutte P. M. (1986): Endothelium-dependent contractions to acetylcholine in the aorta of the spontaneously hypertensive rat. Hypertension 8, 344-348

Martinez-Maldonado M. (1991): Pathophysiology of renovascular hypertension. Hypertension 17, 707-719

Nakamura T., Prewitt R. L. (1992): Alteration of endothelial function in arterioles of renal hypertensive rats at two levels of vascular tone. J. Hypertens. 10, 621-627 
doi:10.1097/00004872-199207000-00004

Nystrom H. C., Jia J., Johansson M., Lambert G., Bergstrom G. (2002): Neurohormonal influences on maintenance and reversal of two-kidney one-clip renal hypertension. Acta Physiol. Scand. 175, 245-251 doi:10.1046/j.1365-201X.2002.00997.x

Panza J. A., Quyyumi A. A., Callahan T. S., Epstein S. E. (1993): Effect of antihypertensive treatment on endothelium-dependent vascular relaxation in patients with essential hypertension. J. Am. Coll. Cardiol. 21, 1145-1151 doi:10.1016/0735-1097(93)90238-V

Parving H. P., Gyntelberg F. (1973): Transcapillary escape rate of albumin and plasma volume in essential hypertension. Circ. Res. 32, 643-651

Paulis L., Matuskova J., Adamcova M., Pelouch V., Simko J., Krajcirovicova K., Potacova A., Hulin I., Janega P., Pechanova O., Simko F. (2008): Regression of left ventricular hypertrophy and aortic remodelling in NO-deficient hypertensive rats: effect of L-arginine and spironolactone. Acta Physiol. (Oxf.) 194, 45-55 doi:10.1111/j.1748-1716.2008.01862.x

Pedrinelli R., Penno G., Dell'Omo G., Bandinelli S., Giorgi D., Di B., V, Nannipieri M., Navalesi R., Mariani M. (1998): Transvascular and urinary leakage of albumin in atherosclerotic and hypertensive men. Hypertension 32, 318-323

Pedrinelli R., Penno G., Dell'Omo G., Bandinelli S., Giorgi D., Di B., V, Navalesi R., Mariani M. (1999): Microalbuminuria and transcapillary albumin leakage in essential hypertension. Hypertension 34, 491-495

Pepine C. J. (2009): The impact of nitric oxide in cardiovascular medicine: untapped potential utility. Am. J. Med. 122, S10-15 doi:10.1016/j.amjmed.2009.03.003

Ross R. (1993): The pathogenesis of atherosclerosis: a perspective for the 1990s. Nature 362, 801-809 doi: $10.1038 / 362801 \mathrm{a} 0$
Ross R. (1999): Atherosclerosis--an inflammatory disease. N.Engl. J.Med. 340, 115-126 doi:10.1056/NEJM199901143400207

Schulman I. H., Zhou M. S., Raij L. (2005): Nitric oxide, angiotensin II, and reactive oxygen species in hypertension and atherogenesis. Curr. Hypertens. Rep. 7, 61-67 doi:10.1007/s11906-005-0056-6

Torok J. (2008): Participation of nitric oxide in different models of experimental hypertension. Physiol. Res. 57, 813-825

van Hinsbergh W. M. (1997): Endothelial permeability for macromolecules. Mechanistic aspects of pathophysiological modulation. Arterioscler. Thromb. Vasc. Biol. 17, 1018-1023

Versari D., Daghini E., Virdis A., Ghiadoni L., Taddei S. (2009): Endothelial dysfunction as a target for prevention of cardiovascular disease. Diabetes Care 32 (Suppl. 2), S314-321 doi:10.2337/dc09-S330

Viazzi F., Leoncini G., Ratto E., Parodi A., Falqui V., Conti N., Tomolillo C., Ravera G., Deferrari G., Pontremoli R. (2008): Vascular permeability, blood pressure, and organ damage in primary hypertension. Hypertens. Res. 31, 873-879 doi:10.1291/hypres.31.873

Yuan Y., Granger H. J., Zawieja D. C., DeFily D. V., Chilian W. M. (1993): Histamine increases venular permeability via a phospholipase C-NO synthase-guanylate cyclase cascade. Am. J. Physiol. 264, H1734-1739

Zhou M. S., Kosaka H., Tian R. X., Abe Y., Chen Q. H., Yoneyama H., Yamamoto A., Zhang L. (2001): L-Arginine improves endothelial function in renal artery of hypertensive Dahl rats. J. Hypertens. 19, 421-429 doi:10.1097/00004872-200103000-00010

Received: October 5, 2010

Final version accepted: January 24, 2011 